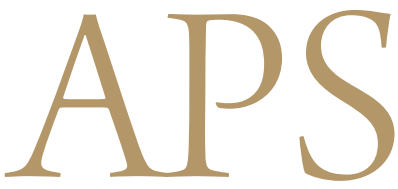

Archives of Plastic Surgery

\title{
Finite element analysis of long-term changes of the breast after augmentation mammoplasty: Implications for implant design
}

\author{
Yujin Myung ${ }^{1 *}$, Jong-Gu Lee ${ }^{2 *}$, Maenghyo $\mathrm{Cho}^{2}$, Chan Yeong $\mathrm{Heo}^{3}$ \\ ${ }^{1}$ Department of Plastic and Reconstructive Surgery, Ajou University Medical Center, Ajou University College of Medicine, Suwon; ${ }^{2}$ Smart \\ Structures and Design Lab, School of Mechanics and Aerospace Engineering, Seoul National University, Seoul; ${ }^{3}$ Department of Plastic and \\ Reconstructive Surgery, Seoul National University Bundang Hospital, Seoul National University College of Medicine, Seongnam, Korea
}

The development of breast implant technology continues to evolve over time, but changes in breast shape after implantation have not been fully elucidated. Thus, we performed computerized finite element analysis in order to better understand the trajectory of changes and stress variation after breast implantation. The finite element analysis of changes in breast shape involved two components: a static analysis of the position where the implant is inserted, and a dynamic analysis of the downward pressure applied in the direction of gravity during physical activity. Through this finite element analysis, in terms of extrinsic changes, it was found that the dimensions of the breast implant and the position of the top-point did not directly correspond to the trajectory of changes in the breast after implantation. In addition, in terms of internal changes, static and dynamic analysis showed that implants with a lower top-point led to an increased amount of stress applied to the lower thorax. The maximum stress values were 1.6 to 2 times larger in the dynamic analysis than in the static analysis. This finding has important implications for plastic surgeons who are concerned with long-term changes or side effects, such as bottoming-out, after anatomic implant placement.

Keywords Mammaplasty / Breast implants / Finite element analysis / Computer simulation
Correspondence: Chan Yeong Heo Department of Plastic and Reconstructive Surgery, Seoul National University Bundang Hospital, Seoul National University College of Medicine, 82 Gumi-ro 173beon-gil, Bundang-gu, Seongnam 13620, Korea Tel: +82-31-787-7222

Fax: +82-31-787-4055

E-mail: lionheo@gmail.com

*The two authors contributed equally to this work.

This research was supported by the Basic Science Research Program through the National Research Foundation of Korea (NRF), funded by the Ministry of Education (2016R1A6A3A11934330).

Received: 23 Mar 2019 • Revised: 16 May $2019 \bullet$ Accepted: 12 Jun 2019

pISSN: 2234-6163 • elSSN: 2234-6171 • https://doi.org/10.5999/aps.2019.00346• Arch Plast Surg 2019;46:386-389

\section{INTRODUCTION}

Major innovations have taken place in implant manufacturing technology for use in cosmetic surgery, followed by an increase in breast cancer reconstructions and the need for aesthetic breast surgery [1]. Although the shape of the breast changes over time during the postoperative period, it is not clear whether these changes depend on the shape of the implant initially implanted [2].
In this study, we investigated how the dimensions of the breast implant, including its projection and height, affect the shape of the breast in the long term. We also investigated the ideal implant dimensions for optimizing breast shape from an aesthetic perspective. In addition to the effects of the dimensions of the breast implant on the morphology of the breast, we analyzed the internal stress on the breast during static and dynamic states after implantation.

In order to analyze the postoperative external shape and inter- 
nal stress on the breast, we simulated an in situ operative breast and a postoperative breast two-dimensionally using a finite element model, which is a widely used method of computational analysis [3].

\section{IDEA}

\section{Finite element modeling}

For two-dimensional finite element analysis (FEA) of the external and internal characteristics of the breast with respect to the initial shape of the breast and its shape after implantation, commercial FEA software was utilized (ABAQUS version 6.12; Dassault Systèmes, Vélizy-Villacoublay, France).

First, as shown in Fig. 1, we characterized the initial shape of a patient's breast and implant in terms of shape parameters such as the projection, top-point, and height. Among the shape parameters, the top-point was selected as the main design parameter because the projection and the arc length change depending on the position of the top-point. Two-dimensional geometric models of the patient's breast and implant were prepared. Additionally, the rigid breast plate, which pushes the implant forward into the breast, was introduced to simulate the process of implant surgery.

Based on the geometric model, a 3-node linear plane stress triangle element (CPS3) was assigned to both the breast and the implant. The finite element model of the implant comprised 430 elements and 245 nodes. For the breast, the finite element model comprised 958 elements and 552 nodes. A 2-node two- dimensional linear rigid link element (R2D2) was assigned to the rigid breast plate. The finite element model of the rigid breast plate comprised 16 elements and 17 nodes.

The implant consisted of a shell and filler, made of polydimethylsiloxane (PDMS) and cohesive silicone gel, respectively. Anatomically, the breast mainly consists of fat tissue and fibroglandular tissue. Generally, compared with implants, breast tissue is much softer and more stretchable, like a rubber material. Thus, the constitutive equations for the breast and implant were derived based on the Mooney-Rivlin [4] model and elasticity model [5], respectively.

\section{Loading and boundary conditions Static analysis of the breast with the implant}

Initially, the implant and the breast were separated from each other, so that the bottom surfaces of the implant were aligned in a direction parallel to the bottom surfaces of the breast without surface overlap. The rigid plate was also separated and placed at the rear of the implant, and aligned parallel to the bottom surface of the implant, as shown in Fig. 1.

\section{Dynamic analysis of the breast with the implant}

Following the static analysis for implantation, the dynamic response of the postoperative breast was estimated by simulating the periodic vibration of the implant-embedded breast, as shown in Fig. 2. For this simulation, the loading and boundary conditions were modified subsequent to implantation.

\section{Fig. 1. Finite element simulation process}

(A) Before implantation of an implant and simulated surface of the implant. (B) Initial encounter between implant and soft tissue. (C) Final result after implantation, showing maximal tension at the lower pole area.

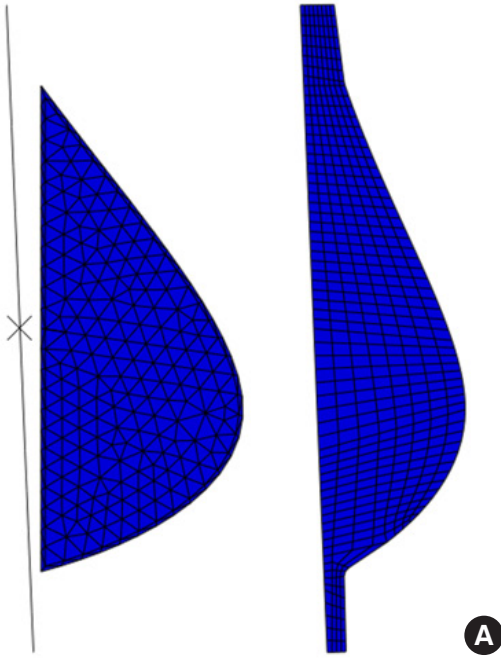

A

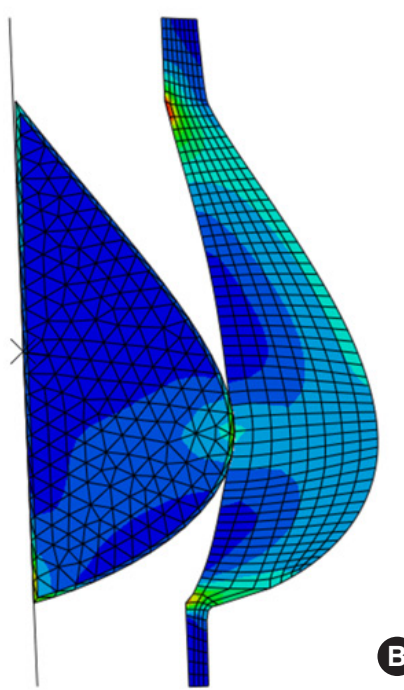

B

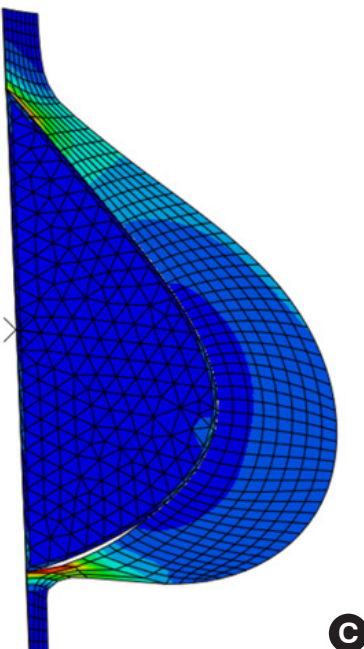




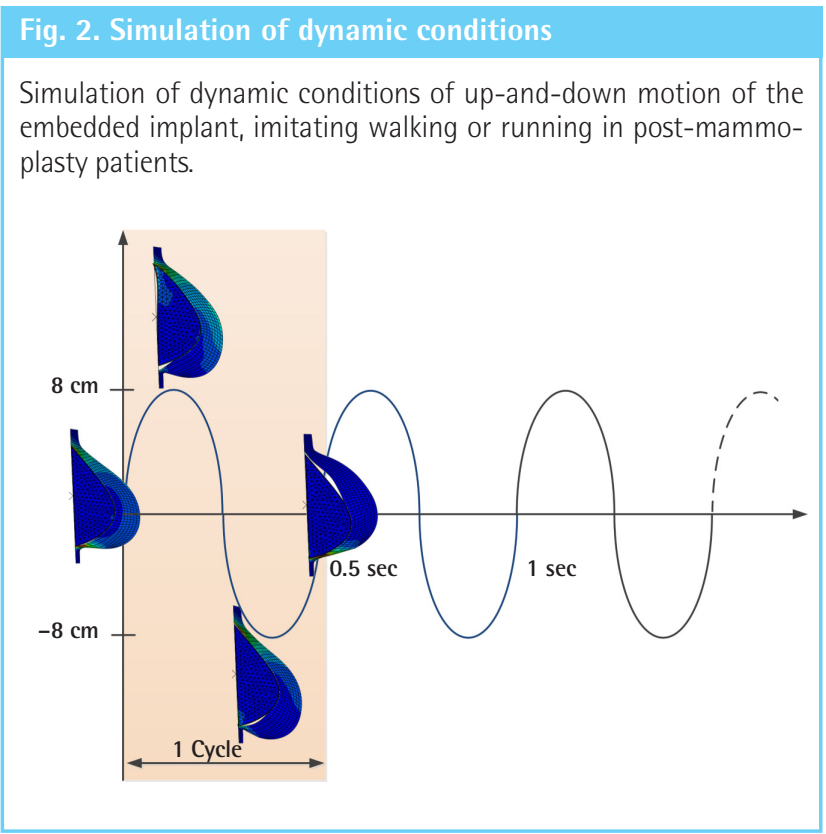

\section{Static analysis}

From the nonlinear static analysis, the extrinsic and intrinsic changes of the breast were analyzed numerically in scenarios involving implants with different top-points. As shown in Fig. 3, the central cross-sectional location of the top-point of the implant-embedded breast tended to increase upward as the toppoint of the implant became lower than the initial top-point of the breast. In contrast, as the top-point became higher, a downward migration was observed.

\section{Dynamic analysis}

The maximum stress alternately appeared in the lower chest and the upper chest during up-and-down dynamic behavior. However, the value of the maximum stress was generally about 1.6 to 2 times greater than the stress level in the static state, as shown in Fig. 4.

\section{DISCUSSION}

Implant-based breast augmentation surgery was first performed more than 40 years ago [6]. Since then, silicone implant technology has been continuously developing, resulting in innovations such as better materials and designs, and the ability to manufacture implants with various dimensions. Although implants with diverse dimensions and designs are used in everyday practice, there have not been any long-term follow-up studies or simulations of morphological changes with different implant shapes.

This study quantitatively estimated the external changes af-

\section{Fig. 3. Different tensive stress between various top points}

Three distinctive features and shapes of implants are shown, with different maximal stress points. The $\mathrm{X}$-axis demonstrates the simulated time period of the breast after initial implantation, and the $Y$ axis demonstrates the relative location of the maximal projection point of the implanted breast. An anatomical implant with a lower top-point showed maximal tensive stress on the lower pole of the breast.

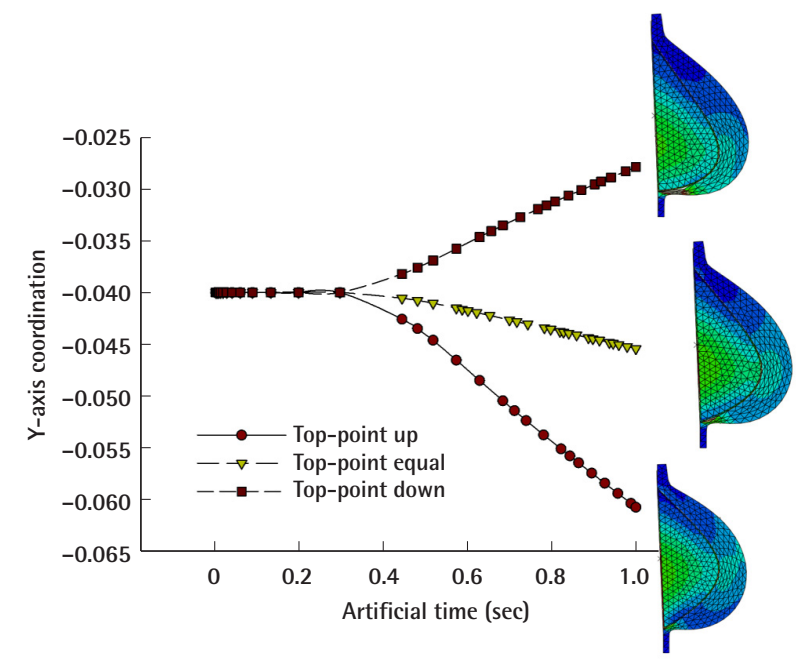

Fig. 4. Stress in static and dynamic simulations

Comparison of the amount of stress in static and dynamic simulations between different implant shapes. An anatomic implant with a lower top-point yielded more quantitative stress during periodic motion of the implant-embedded breast.

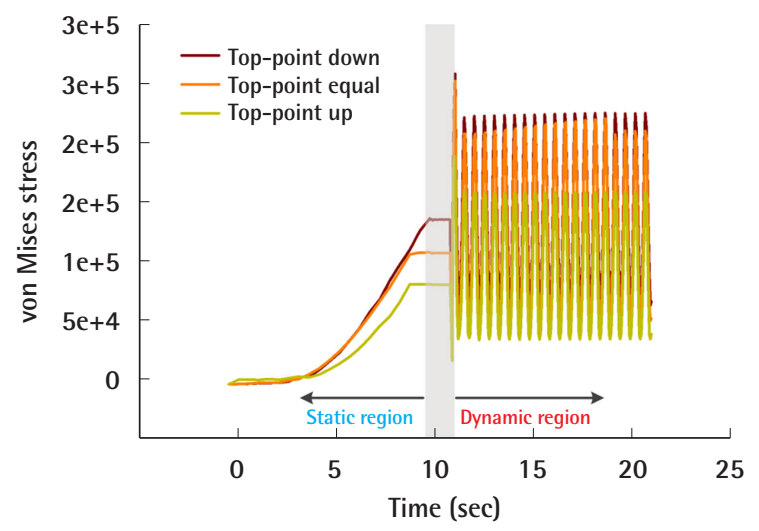

fecting postoperative breast aesthetics according to the height of the breast, the breast implant, and the relative position of the protrusion. In particular, internal characteristics such as mechanical stress showed significant quantitative differences depending on the shape of the breast implant and its location in relation to the chest. In particular, stress was maximized at the lower part of the chest, which is causally related to the clinical manifestation of inframammary fold tear, which most often oc- 
curs in the lower chest.

In the present study, it was shown that the mechanical stress applied to the lower chest region varied according to the maximal projection point of the breast implant. The maximum stress value received by the lower chest area was 1.6 to 2 times larger during dynamic motion than in static conditions.

The study has several limitations. First, it was performed using two-dimensional analysis, rather than a three-dimensional simulation, which would have been helpful for a more precise analysis. However, the most intriguing aspect of this study is the change in the central cross-sectional shape between the breast implant and the soft tissue envelope of the breast in the sagittal section after insertion of the implant. Based on the fact that the compression under the human chest after implant insertion (referred to as stress in this study) is also greatest near the bottom of the central section of the breast implant, we hypothesize that a two-dimensional study in a central cross-sectional plane may be more accurate for a static and dynamic analysis of pressure measurements in the central section.

Additionally, the location of the inframammary fold was fixed at a single point, rather than migrating in the downward direction, as in actual practice. However, because the goal of this study was to establish a boundary using a reference point for rigid plate alignment in terms of FEA, and to perform quantitative measurements using stress analysis on this boundary, it was not also possible to measure changes in the position of the inframammary fold, for which additional, separately designed research would be required.

\section{NOTES}

\section{Conflict of interest}

No potential conflict of interest relevant to this article was reported.

\section{Author contribution}

Conceptualization: Myung Y, Lee JG, Heo CY. Data curation: Lee JG. Formal analysis: Lee JG, Cho M. Methodology: Myung Y, Lee JG. Project administration: Cho M, Heo CY. Visualization: Lee JG. Writing - original draft: Myung Y, Lee JG. Writing - review \& editing: Myung Y, Lee JG. Approval of final manuscript: all authors.

\section{ORCID}

Yujin Myung https://orcid.org/0000-0001-5051-2440

Jong-Gu Lee https://orcid.org/0000-0003-2278-112X Maenghyo Cho https://orcid.org/0000-0003-3942-9261

Chan Yeong Heo https://orcid.org/0000-0001-9003-7365

\section{REFERENCES}

1. Rocco N, Rispoli C, Moja L, et al. Different types of implants for reconstructive breast surgery. Cochrane Database Syst Rev 2016; (5):CD010895.

2. Rowe NM, Freund R. The autologous internal breast splint: a novel technique for the treatment of postaugmentation ptosis. Aesthet Surg J 2005;25:587-92.

3. Georgii J, Eder M, Burger K, et al. A computational tool for preoperative breast augmentation planning in aesthetic plastic surgery. IEEE J Biomed Health Inform 2014;18:907-19.

4. Pianigiani S, Ruggiero L, Innocenti B. An anthropometricbased subject-specific finite element model of the human breast for predicting large deformations. Front Bioeng Biotechnol 2015;3:201.

5. Rynkevic R, Martins P, Parente M, et al. Implant shape influence on the mechanical behavior of breast implants. Presented at 2013 IEEE 3rd Portuguese Meeting in Bioengineering (ENBENG); 2013.

6. De Cholnoky T. Present status of augmentation mammaplasty. Conn Med 1970;34:808-9. 